\title{
Triggered massive star formation in the open cluster NGC 1893
}

\author{
Amparo Marco and Ignacio Negueruela \\ Departamento de Física, Ingeniería de Sistemas y Teoría de la Señal, \\ Univ. de Alicante, Apdo. de Correos 99, E-03080 Alicante, España
}

\begin{abstract}
We have carried out a search for emission line pre-main sequence (PMS) stars in the area of the star-forming young open cluster NGC 1893 by means of slitless spectroscopy. We find 19 emission line stars with spectral types spanning the range from early B-type to G-type. All the PMS stars are confined to two small regions: the outer rim of the molecular cloud associated with the $\mathrm{H}$ II region IC 410 and the vicinity of the bright emission cometary nebulae Sim 129 and Sim 130. The overall spatial distribution of PMS stars strongly suggests, that present day star formation in NGC 1893 is triggered by the O-type stars in the cluster.
\end{abstract}

\section{Introduction}

NGC 1893 appears as a very extended region of loosely grouped early-type stars, associated with the H II region IC 410 and obscured by several conspicuous dark clouds. The cluster is located at a distance of $6 \mathrm{kpc}$ and very moderately reddened (Marco et al. 2001). NGC 1893 contains five O-type stars and its B-type stars fit well to the ZAMS down to $\sim$ B6. In spite of this, several massive ( B1) Herbig Be pre-main sequence stars are present, indicating that massive stars have not formed coevally. In recent work, we have obtained Strömgren photometry of over 40 MS members and located several PMS candidates (Marco et al. 2001; Marco \& Negueruela 2002).

\section{Observations}

Slitless spectroscopy was obtained during the nights of $5-7$ December 2001 with ALFOSC on the 2.6-m Nordic Optical Telescope (NOT) in La Palma. Slit spectroscopy of several PMS and MS stars has been taken during different runs at the 2.5-m Isaac Newton Telescope (INT, in La Palma), NOT and 1.93-m Telescope at the Haute-Provence Observatory.

We also obtained $\mathrm{H} \alpha$ imaging of the area using the wide Field Camera on the INT, on 11th December 2001.

\section{Results}

We observed five frames covering a large area of NGC 1893 with slitless spectroscopy. We find 19 emission line stars in the region of NGC 1893. The detection 
limit depends strongly on how crowded a particular field is, but we estimate that our sample is complete down to $V>17$ and we detect some faint emission line objects with $V \approx 19$ (corresponding to the intrinsic magnitude of a MS F-type star in NGC 1893).

The spectral types of several emission-line objects have been derived from long-slit spectra. They cover the range from B1 to early G-type, but the spectral distribution differs considerably from a standard IMF, with five stars displaying B0.5 - B2 spectral types. Most of the fainter objects are of spectral F-type.

PMS stars concentrate in two small regions of the cluster. Towards the cluster core, we find six emission-line PMS stars lying in the vicinity of two O-type stars and several B-type MS members, close to the the outer rim of the molecular cloud associated with the H II region IC 410. One of this objects has a spectral type B0.5e.

Towards the NE, we find two bright emission pennant nebulae, Sim 129 and Sim 130. In their immediate vicinity, we find 13 PMS emission-line stars and a few B-type objects, which seem to have already reached the ZAMS. Four of the PMS stars have spectral types B1-B2. In addition, the $\mathrm{H} \alpha$ image clearly shows the presence of evaporating gaseous globules on the outer rims of both Sim 129 and Sim 130. This is an area of intense star formation.

\section{Discussion}

The area where emission-line stars are present represents a very small fraction of the region surveyed. In contrast, the area to the East and SE of the molecular cloud, where most MS members lie, is devoid of emission line stars. Of particular interest is the fact that the most luminous star in the cluster, the $04 \mathrm{~V}((\mathrm{f}))$ star HD 242908, lies in a region relatively devoid of cluster members. The $\mathrm{H} \alpha$ image shows that this star has created a vast cavity in the surrounding molecular cloud.

The resulting picture seems to be in good agreement with the standard model of star formation in a molecular cloud. The O-type stars in NGC 1893 have evacuated a vast cavity in the molecular cloud because of their powerful UV winds, and triggered star formation is taking place on the illuminated rims of the surviving cloud. It must be noted, however, that a significant population of early-type B stars lies further East from IC 410 than the area surveyed. Before the spatial structure of this star forming region can be fully understood, detailed investigation of a very large area $\left(\sim 45^{\prime} \times 45^{\prime}\right)$ will be necessary.

Acknowledgments. Some of the long-slit spectra were obtained with the INT as part of the Isaac Newton Group service programme. We thank G. Bernabeu for his help during an INT run and G.L. Israel for his help during the NOT observations.

\section{References}

Marco, A., Bernabeu, G., Negueruela, I. 2001, AJ 121, 2075

Marco, A., Negueruela, I. 2002, A\&A 393, 195 